\title{
Percepción de amenaza como mediadora de la relación entre los estereotipos y el prejuicio hacia los migrantes venezolanos en Perú
}

\author{
Perception of threat as a mediator of the relationship between stereotypes and prejudice \\ towards Venezuelan migrants in Peru
}

\author{
Milagros Gomez Robinson" ${ }^{\mathrm{a}, *}$, Agustín Espinosa ${ }^{\mathrm{a}}$ \\ aPontificia Universidad Católica del Perú, Perú
}

\begin{abstract}
Resumen
Objetivos: el presente estudio tiene como objetivo explorar la relación entre los estereotipos (moralidad, calidez y competencia) y el prejuicio (sutil y manifiesto) hacia los inmigrantes venezolanos en Perú. Además, se analiza el rol mediador de la percepción de amenaza en la relación entre los estereotipos y los prejuicios hacia este grupo social. Método: se realizó un diseño de investigación correlacional, utilizando la Escala de Prejuicio Sutil y Manifiesto, la Escala de Estereotipos Nacionales y la Escala de Percepción de Amenaza Exogrupal. La muestra consiste en 115 participantes de nacionalidad peruana de entre 18 y 60 años $(M=29.6 ; D E=11.77)$. Resultados: los resultados confirman la relación inversa entre la representación positiva y el prejuicio ambivalente, es decir, cuando los venezolanos son representados como más competentes, cálidos y morales, se aprecian menos expresiones de prejuicio sutil y manifiesto hacia este grupo social. El coeficiente de regresión de las relaciones entre estereotipos y prejuicios tiende a disminuir cuando la amenaza percibida es incorporada como variable mediadora al modelo de mediación parcial. Conclusiones: se confirman las relaciones entre las variables estudiadas. Además, se discute su relevancia para la implementación de intervenciones que promuevan el contacto intergrupal, la inclusión social, y el cuestionamiento de agentes sociales que refuerzan una representación amenazante del inmigrante latinoamericano.
\end{abstract}

Palabras clave: migración venezolana; estereotipos; prejuicio; percepción de amenaza.

\section{Para citar este artículo:}

Gomez, M., \& Espinosa, A. (2021). Percepción de amenaza como mediadora de la relación entre los estereotipos y el prejuicio hacia los migrantes venezolanos en Perú. Liberabit, 27(1), e451. https:/ /doi.org/10.24265/liberabit.2021.v27n1.04

\begin{abstract}
Objectives: The present study aims to explore the relationship between stereotypes (morality, warmth, and competence) and prejudice (subtle and blatant) towards Venezuelan immigrants in Peru. In addition, the role of threat perception as a mediator in the relationship between stereotypes and prejudice towards this social group is analyzed. Method: A correlational research design was conducted, using the Subtle and Blatant Prejudice Scale, the National Stereotypes Scale, and the Out-Group Threat Perception Scale. The sample consisted of 115 participants of Peruvian nationality between 18 and 60 years old $(M=$ 29.6; $S D=11.77$ ). Results: The results confirm the inverse relationship between positive representation and ambivalent prejudice. In other words, when Venezuelans are represented as more competent, warm and moral, there are fewer expressions of subtle and blatant prejudice towards this social group. The regression coefficient of the relationships between stereotypes and prejudice tends to decrease when perceived threat is incorporated as a mediating variable in the partial mediation model. Conclusions: The relationships between the studied variables are confirmed. Furthermore, their importance for the implementation of interventions that promote intergroup contact, social inclusion, and questioning of social agents that reinforce a threatening representation of the Latin American immigrant is discussed.
\end{abstract}

Keywords: Venezuelan migration; stereotypes; prejudice; threat perception.

Este es un artículo Open Access publicado bajo la licencia Creative Commons Atribución 4.0 Internacional. (CC-BY 4.0)

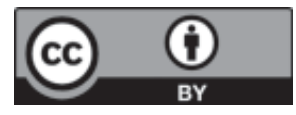

Universidad de San Martín de Porres, Lima - Perú

* milagros.gomez@pucp.pe 


\section{Introducción}

Las migraciones son fenómenos sociales e históricos de gran incidencia, que comprenden el desplazamiento de grandes grupos humanos, cuyos miembros deciden movilizarse o son forzados a hacerlo desde una sociedad de origen hacia una sociedad de acogida o destino. Los procesos migratorios se originan por motivos de seguridad, económicos y sociales que influyen en las condiciones y calidad de vida de las personas en un mundo cada vez más interconectado (Lotero-Echeverri \& Pérez-Rodríguez, 2019; Organización Internacional para las Migraciones [OIM], 2018). Según el último registro de la OIM, al año 2017 se estima que existen 257.7 millones de migrantes internacionales, lo que equivale al 3.4\% de la población mundial (OIM, 2018). De este grupo, las mujeres constituyen el 48\% de la población migrante; asimismo, habría aproximadamente 36.1 millones de niños, niñas y adolescentes en esta situación, 4.4 millones de estudiantes internacionales y 150.3 millones de migrantes por cuestiones laborales.

Entre las causas de las migraciones internacionales, destacan las crecientes brechas entre los países de origen y de destino en el nivel de renta, las oportunidades diferenciadas de acceso a bienes y servicios, la calidad de vida y la garantía del disfrute de derechos ciudadanos en general (Lotero-Echeverri \& Pérez-Rodríguez, 2019; OIM, 2018). Además, en los últimos años se ha evidenciado un aumento de los desplazamientos provocados por conflictos, persecuciones, situaciones de degradación y cambio ambiental, la reestructuración de la producción y demanda de mano de obra barata, el rápido crecimiento demográfico y la falta de oportunidades y seguridad, entre otras razones (Meneses, 2012; OIM, 2018).

Los flujos migratorios de una sociedad a otra generan situaciones de contacto intergrupal; es decir, producen dinámicas intergrupales en la interacción entre los extranjeros y los ciudadanos del país receptor, donde la migración de grupos humanos en búsqueda de mejores condiciones de vida supone, frecuentemente, su posicionamiento en grupos sociales minoritarios, de bajo estatus y escaso acceso al poder (Contreras-Ibáñez \& Saldívar, 2018; Mera et al., 2014). Según Martínez (1996), lo determinante en las relaciones intergrupales es la existencia de reciprocidad o antagonismo en los intereses de los grupos. Cuando los recursos son supuestamente escasos y un grupo compite con otro para satisfacer sus necesidades o lograr ventajas materiales, la competencia económica genera controversias donde el prejuicio se muestra como instrumento de lucha y justificación de comportamientos hostiles hacia los migrantes.

La presencia de migrantes desencadena procesos cognitivos, actitudinales y comportamentales en el grupo de personas que habitan el país receptor. En otras palabras, se construyen estereotipos, se manifiestan prejuicios y se pueden presentar diversas conductas discriminatorias. Los estereotipos son comúnmente definidos como los rasgos y las características considerados representativos de los grupos sociales, de los miembros de dichos grupos y, particularmente, comprenden aquellos atributos que permiten diferenciar a los grupos entre sí (Stangor, 2016). Cuando se activa un estereotipo, se recuerdan los conocimientos, las creencias y las expectativas acerca de un grupo (Sherman, 1996). Si bien existen estereotipos positivos, los estereotipos sobre los exogrupos y los grupos desventajados tienden a tener más connotaciones negativas que los estereotipos sobre los endogrupos y los grupos privilegiados (Castro, 2006).

Las evaluaciones situacionales de posibles daños o beneficios en la interacción intergrupal contienen creencias estereotípicas que, usualmente, se construyen a partir de dos dimensiones: calidez y competencia. Los estereotipos de calidez se relacionan con características orientadas a las relaciones sociales, por lo cual, ayudan a anticipar las intenciones de otros hacia el endogrupo. Mientras que los estereotipos de competencia se relacionan con características orientadas a la tarea, la eficacia y el intelecto, por lo 
tanto, ayudan a reconocer la capacidad de los demás para lograr sus objetivos (Fiske, 2000; López-Rodríguez et al., 2013; Yzerbyt, 2016).

Otros estudios complementan el modelo mencionado con la incorporación de una tercera dimensión: la moralidad. Esta incluye rasgos como la honestidad, la solidaridad y la tolerancia (Brambilla et al., 2012; Fiske et al., 2002; Phalet \& Poppe, 1997). Según Wojciszke (1994), en el rol de agencia, las personas suelen interpretar su propio comportamiento en términos de competencia, pero como observadores lo interpretan en categorías morales (Moscatelli et al., 2018; Phalet \& Poppe, 1997). En países latinoamericanos, se encuentra que la moralidad puede ejercer efectos positivos tanto en la autoestima colectiva como en el grado de identificación (Espinosa et al., 2016). En general, la dimensión moral influye en la interacción entre grupos, las expectativas sobre el exogrupo y contribuye a identificar relaciones intergrupales agresivas, cooperativas, competitivas o altruistas.

Con respecto al prejuicio, su definición comprende una actitud negativa en contra de un objeto o categoría social relacionada con una creencia estereotípica, sobregeneralizada y errónea acerca del objeto o categoría social en cuestión (Allport, 2000). Tradicionalmente, el prejuicio busca explicarse desde sus componentes cognitivos y afectivos, donde el carácter actitudinal del mismo está acompañado por una racionalización implícita que es difícil de modificar (Allport, 2000; Stangor, 2016). En mayor o menor medida, puede ser un factor preliminar del comportamiento, en tanto establece y sostiene las condiciones óptimas para la existencia de conductas discriminatorias.

En la formación de creencias y actitudes que justifican la discriminación, surgen dos expresiones de prejuicio: manifiesto y sutil. El prejuicio manifiesto es cercano y directo, representa el rechazo extremo e intolerante, y está fundamentado en la percepción de superioridad genética del propio grupo y por la defensa de la segregación social de los grupos considerados subordinados (Pettigrew \& Meertens, 1995; Rueda \& Navas, 1996). Además, involucra el rechazo del exogrupo porque se le percibe amenazante, lo cual se expresa en la oposición al contacto íntimo o al trabajo bajo la supervisión de alguno de sus miembros (Pettigrew \& Meertens, 1995).

En cambio, el prejuicio sutil es distante e indirecto, oculta una profunda intolerancia a la diversidad cultural y rechaza a ciertos grupos porque se les acusa de provocar mayor inseguridad ciudadana y generar mayor competencia económica en la sociedad (Pettigrew \& Meertens, 1995; Rueda \& Navas, 1996). Además, las expresiones de prejuicio sutil suelen ser consideradas normativas y aceptables al involucrar la defensa de valores tradicionales, la exageración de las diferencias culturales y el rechazo hacia las respuestas emocionales positivas hacia el exogrupo (Pettigrew \& Meertens, 1995; Olson \& Zabel, 2016). En general, las expresiones de prejuicio sutil ocultan una arraigada intolerancia a la diversidad cultural y pueden transformarse en conductas racistas cuando la circunstancia social lo propicia, por lo tanto, el prejuicio manifiesto no se presenta de forma aislada, pues tiene a la base creencias y actitudes sutiles de prejuicio (Rueda \& Navas, 1996).

La población inmigrante es percibida como competitiva cuando se asocia al disfrute de recursos sociales y amenazante cuando se relaciona con la inseguridad ciudadana o la preservación de la identidad del endogrupo (Martínez et al., 2011). Según Stephan y Stephan (2000), a partir de las amenazas contra la integridad endogrupal, se generan expresiones de prejuicio hacia los grupos culturalmente diferentes. La amenaza puede derivarse de una de las cuatro fuentes del denominado Modelo de Amenaza Integrado: amenaza real, amenaza simbólica, ansiedad intergrupal y estereotipos negativos (Willis-Esqueda et al., 2017). La amenaza real se refiere al daño contra la existencia, el poder político y económico del endogrupo, así como del bienestar físico y material 
de sus miembros. La amenaza simbólica implica el desafío a la moral, los valores, las creencias y la cultura del endogrupo. De hecho, el modelo enfatiza que las amenazas realistas y simbólicas no tienen que ser reales para afectar las actitudes intergrupales, y por ende la expresión del prejuicio (Ríos et al., 2018). La ansiedad intergrupal es la inquietud que uno experimenta cuando interactúa con otro grupo o alguno de sus miembros. Por último, los estereotipos negativos son las creencias o respuestas evaluativas hacia alguna persona o grupo (Stephan et al., 1999). La premisa central de este modelo es que estas cuatro formas de amenaza predicen de forma independiente los prejuicios de los miembros de un grupo hacia los miembros de otro (Ríos et al., 2018; Stephan \& Stephan, 2000).

En el contexto de recepción masiva de inmigración extranjera y crisis económica, las nuevas formas de prejuicio y la activación de la percepción de amenaza exogrupal pueden determinar las respuestas ante las minorías inmigrantes (Wlodarczyk et al., 2014). En específico, se encuentra que la valoración y representación mental de los miembros del endogrupo, sobre el mismo, influyen las respuestas afectivas y actitudinales hacia el exogrupo. Como lo evidencian Mackie et al. (2000), en un estudio cuyo objetivo fue investigar la naturaleza y el impacto de la evaluación intergrupal, se encontró que los participantes que perciben al endogrupo como fuerte y organizado, son más propensos a reportar emociones hostiles (p.e., ira y miedo) y querer tomar acciones contra el exogrupo, como oponerse y provocar enfrentamientos o discusiones.

En este sentido, es pertinente discutir el rol de distintos actores y situaciones sociales en la evaluación intergrupal. Se encuentra evidencia sobre el impacto de los medios de comunicación y las campañas políticas en la difusión de estereotipos y prejuicios asociados a la hostilidad contra los migrantes. A diario los medios de comunicación difunden reportajes sobre diferentes aspectos de la migración, principalmente sobre los aspectos negativos (OIM, 2018). Se ha encontrado que la hostilidad contemporánea contra los inmigrantes se basa en estereotipos hacia grupos de inmigrantes particulares y su representación en los medios de comunicación, por lo tanto, el papel de los medios suele ser negativo al influir indirectamente en las creencias y actitudes, a través de las percepciones de amenaza (Atwell et al., 2018; Hainmueller \& Hopkins, 2014; Schlueter \& Davidov, 2013). Además, la migración es objeto de especial interés por parte de actores políticos. A nivel mundial, culpar a los migrantes irregulares de generar problemas sociales se ha convertido en una práctica ampliamente difundida por políticos populistas (Castles, 2010).

\section{La migración venezolana en el Perú}

Debido al contexto socioeconómico y político de Venezuela, la movilización de sus ciudadanos se ha incrementado considerablemente. Dentro de los múltiples destinos elegidos por los migrantes venezolanos, Perú ocupa el segundo lugar en América Latina (Granados, 2019). Según la Superintendencia Nacional de Migraciones (SNM, 2018), desde el 2014 al 2016 ingresaron al país alrededor de $50 \mathrm{mil}$ migrantes venezolanos por año, para el 2017 esta cifra se incrementó a más de 223 mil y, según su más reciente registro, para el 2019 la cifra se incrementó a 750 mil.

Para los ciudadanos peruanos, la llegada masiva de inmigrantes provenientes de un país latinoamericano es un fenómeno reciente y novedoso en el contexto contemporáneo. Esto al considerar que el Perú ha sido tradicionalmente un país de emigrantes debido a las condiciones sociales del país durante las últimas décadas del siglo XX. Es importante analizar la transición del Perú, de un país en crisis, a un país que resulta atractivo en la región sudamericana para desarrollar y potenciar las oportunidades de desarrollo del migrante venezolano. Frente a estos cambios en la propia conducta migratoria de los peruanos es interesante observar que, ante la llegada de ciudadanos extranjeros, se ha propiciado la exposición de 
elementos sociales y psicológicos que son resultado de una sociedad de acogida que aparentemente no está social y psicológicamente preparada para recibir a otras personas.

A nivel nacional, se ha encontrado evidencia que retrata la desaprobación de la migración por parte de los peruanos. En una encuesta realizada por Ipsos en colaboración con El Comercio, se encuentra que dos de cada tres limeños (67\% de la población de Lima) desaprueba la inmigración de ciudadanos venezolanos. Entre los argumentos esgrimidos, se encuentran que los venezolanos disminuyen la oferta laboral para peruanos, incrementan la informalidad, generan conflictos sociales y aumentan las tasas de criminalidad (Alayo, 2019; Instituto de Opinión Pública [IOP], 2018). Sin embargo, otras evidencias demuestran que, aunque la mayoría de inmigrantes venezolanos presenta dificultades para convalidar sus estudios y obtener un permiso legal para incorporarse al mercado laboral, se calcula que para 2018 y 2019 la presencia de inmigrantes venezolanos ha incrementado el PBI del país en un .08\% (Perea et al., 2019).

Por lo tanto, si tomamos en cuenta el impacto positivo que la migración venezolana puede tener en la economía peruana, es relevante cuestionar qué otros aspectos pueden verse influenciados por la presencia de los inmigrantes. En este sentido, la interacción intergrupal podría beneficiar la construcción de representaciones positivas sobre el inmigrante, las actitudes vinculadas al mismo y la mitigación de conductas discriminatorias. Considerando la deficiente respuesta institucional para la recepción de migrantes, la falta de políticas de integración y el abordaje sesgado de los medios de comunicación, es pertinente concluir que no se han brindado las condiciones mínimas o ideales para la adecuada integración de los migrantes venezolanos en el país (Arana \& Espinosa, 2020).

Es en base a la aproximación conceptual y la descripción del contexto de la inmigración venezolana en el Perú que se proponen los siguientes objetivos de investigación: (1) analizar las relaciones entre los estereotipos de competencia, calidez y moralidad con el prejuicio ambivalente hacia la migración venezolana en peruanos que residen en Lima; y (2) analizar el rol mediador de la percepción de amenaza exogrupal en la relación entre estereotipos de competencia, calidez y moralidad con el prejuicio ambivalente hacia la migración venezolana en la misma muestra. De acuerdo a las hipótesis planteadas, se espera encontrar que, a mayor estereotipia positiva de competencia, calidez y moralidad hacia los inmigrantes, se presenten menores expresiones de prejuicio en las dimensiones manifiesta y sutil. Asimismo, consideramos que la relación entre estereotipos y prejuicio va a ser mediada por la amenaza exogrupal percibida, basándonos en que la interacción con el exogrupo proveniente de Venezuela está influenciada por expresiones y actitudes negativas debido a que se considera que amenazan los valores, la seguridad ciudadana y los derechos básicos, encontrándose que la percepción de amenaza predecirá el prejuicio ambivalente hacia el exogrupo venezolano (Pereira et al., 2009; Wlodarczyk et al., 2014).

\section{Método}

\section{Participantes}

Para ejecutar el diseño de investigación correlacional, se identificó a los participantes mediante el muestreo aleatorio simple. La muestra estuvo conformada por 115 participantes de nacionalidad peruana que residían en la ciudad de Lima al momento de la aplicación del cuestionario. Como criterios de inclusión a la muestra, se consideró que los participantes sean peruanos de nacimiento, mayores de edad y que residan en algún distrito limeño. La edad promedio fue 29.6 años $(D E=11.77)$ y el rango de edades de los participantes fue de 18 a 60 años. La muestra estuvo conformada, en su mayoría, por mujeres (73\%). El $37.4 \%$ de los participantes residen en distritos en Lima-Centro y el $22.6 \%$ en Lima-Centro-Sur. El $66.1 \%$ de la muestra tiene estudios universitarios de pregrado y 
el 73.9\% se ubica en el nivel socioeconómico medio. Con respecto a la ocupación de los participantes, el $56.5 \%$ son estudiantes, el $19.1 \%$ son trabajadores asalariados, y el $14.8 \%$ trabajan de forma independiente. Se consultó por la experiencia migratoria propia y de familiares, donde el $63.5 \%$ respondió que no ha migrado por estudios ni trabajo, aunque el $81.7 \%$ reporta tener familiares viviendo en el extranjero.

\section{Instrumentos}

Ficha sociodemográfica. Se utilizó una ficha sociodemográfica cuyo objetivo fue identificar el sexo, la edad, el distrito de residencia, el nivel socioeconómico percibido, la ocupación de los participantes considerando seis opciones (estudiante, empleador, asalariado, trabajador familiar no remunerado, trabajador del hogar o trabajador independiente), el grado de instrucción y si se ha tenido alguna experiencia migratoria por estudios o trabajo, si algún familiar ha migrado al extranjero, si ha tenido contacto con los migrantes venezolanos, el grado del contacto con migrantes venezolanos considerando cuatro escenarios (conocidos, amigos, vecinos o familiares) y el grado de similitud percibido entre peruanos y migrantes venezolanos.

\section{Escala de Percepción de Amenaza} Exogrupal (EPAE, Navas et al., 2012). La cual consta de 12 ítems, con una consistencia interna de $\alpha=.95$, considerando dos dimensiones de amenaza: realista (8 ítems, $\alpha=.95$ ) y simbólica (4 ítems, $\alpha=$ .91). Los participantes deben responder utilizando un formato de respuesta tipo Likert de 5 puntos (1 = nada, 5 = mucho), e indicar el grado en que sienten que están en peligro los valores educativos, las creencias religiosas, el acceso a un puesto de trabajo, al sistema sanitario, entre otros, debido a la presencia de un exogrupo específico (Carmona-Halty et al., 2018). La presente escala ha sido previamente adaptada a la población objetivo (Carmona-Halty \& Navas, 2016; López-Rodríguez et al., 2020). Este modelo presentó una adecuación muestral relevante en el análisis factorial exploratorio, $\mathrm{KMO}=.915, p<.001$, en el cual se obtuvo dos dimensiones que explican el $77.43 \%$ de la varianza total.

Escala de Estereotipos Nacionales (adaptado por Espinosa et al., 2016). La escala consta de 24 ítems que comprenden una lista de adjetivos positivos y sus antónimos, de modo que caracteriza qué tanto estos atributos son representativos del exogrupo evaluado. Esta escala presenta una consistencia interna de $\alpha=.96$. Los participantes respondieron un formato de respuesta de 7 puntos, donde 1 representa el acuerdo de que los miembros del exogrupo presentan el atributo negativo, y 7 representa el acuerdo de que presentan el atributo positivo. Cabe agregar que se incluyeron 5 ítems nuevos a la escala original: arrogantes/soberbioshumildes/sencillos en la dimensión de calidez, vulnerables/débiles-invulnerables/fuertes en la dimensión de competencia, y sucios-limpios, peligrosos-no peligrosos y desagradecidosagradecidos en la dimensión de moralidad. En el presente estudio la escala fue sometida a un análisis factorial exploratorio en el que se obtuvo tres factores: calidez ( $\alpha=.88)$, competencia $(\alpha=.93) \mathrm{y}$ moralidad $(\alpha=.89)$. El modelo presenta una adecuación muestral notable en el análisis factorial exploratorio, $\mathrm{KMO}=.923, p<.001$, dichas dimensiones explican el $67.89 \%$ de la varianza total.

Escala de Prejuicio Sutil y Manifiesto (adaptado por Pettigrew \& Meertens, 1995; validado en Argentina por Muller et al., 2017). La escala está compuesta por 12 ítems con una consistencia interna de $\alpha=.86$, que determinan el prejuicio manifiesto (5 ítems, $\alpha=.81$ ) y el prejuicio sutil (7 ítems, $\alpha=.72$ ). El formato de respuesta de la escala fue tipo Likert de 5 puntos $(1=$ totalmente en desacuerdo, $5=$ totalmente de acuerdo). Los ítems de la dimensión de prejuicio sutil deben invertirse para ser interpretados. La presente escala ha sido anteriormente utilizada en la población objetivo (Palacio et al., 2020; Civalero et al., 2019). El modelo presenta una adecuación muestral aceptable en el 
análisis factorial exploratorio, $\mathrm{KMO}=.863, p<.001$, en el cual se obtuvo dos dimensiones que explican el $52.26 \%$ de la varianza total.

\section{Procedimiento}

Para el levantamiento de información, los cuestionarios utilizados fueron digitalizados y adaptados a la plataforma de Google Forms. En la primera parte del formulario se presentó el consentimiento informado, el cual menciona el objetivo del estudio, los criterios de inclusión y las condiciones de confidencialidad y voluntariedad del proceso. Una vez que las personas dieran su consentimiento a participar del estudio, se desplegaba la ficha de datos sociodemográficos y los cuestionarios para la medición de los constructos descritos en el acápite de medición. Los participantes fueron contactados virtualmente mediante redes sociales. Se compartieron publicaciones recurriendo a redes de contacto y grupos virtuales para encontrar la cantidad necesaria de participantes. Cabe agregar que la aplicación se inició en el mes de junio del año 2019.

\section{Análisis de datos}

En primer lugar, para los análisis estadísticos se realizaron análisis de consistencia interna de las escalas utilizadas mediante el coeficiente alfa de Cronbach y los análisis de validez. A continuación, se procesaron los estadísticos descriptivos de las variables del estudio. Posteriormente, se realizaron análisis de correlaciones y regresiones lineales simples, así como, los análisis de mediación correspondientes a los objetivos de investigación propuestos.

\section{Resultados}

\section{Análisis descriptivos y de comparación de medias}

El $40.9 \%$ de los participantes de la muestra considera que tiene poco contacto con los migrantes venezolanos, mientras que el $27 \%$ asegura que no tiene ningún contacto con los migrantes venezolanos. El 73\% de participantes dice que tiene al menos un conocido que haya migrado de Venezuela. Más del $50 \%$ de participantes tiene al menos un vecino que es migrante venezolano. Los participantes que no tienen compañeros de trabajo venezolanos representan el $74.8 \%$ de la muestra. El 35.7\% considera que tiene al menos un amigo que haya migrado de Venezuela. No obstante, los participantes que no tienen familiares representan el $87.8 \%$ de la muestra. Con respecto al grado de similitud entre peruanos y venezolanos, el $40 \%$ considera que son poco parecidos, el $29.6 \%$ cree que son algo parecidos y el $13 \%$ que no se parecen en nada a los peruanos. Sin embargo, el $17.4 \%$ considera que peruanos y venezolanos se parecen mucho.

En la Tabla 1, se presenta el análisis descriptivo de las variables de estudio planteadas. A nivel narrativo, los participantes perciben ordinalmente a los venezolanos como moderadamente cálidos, competentes y morales. Asimismo, las expresiones del prejuicio sutil suelen ser mayores que las del prejuicio benevolente, aunque ambas puntuaciones se ubican por debajo del punto medio de la escala de evaluación. Algo similar ocurre con la amenaza percibida en el exogrupo venezolano que resulta medio baja considerando el punto medio de la escala de respuesta. Igualmente, la amenaza simbólica percibida es menor que la amenaza real.

\section{Análisis de correlación entre variables del estudio}

Se analizó la relación entre los datos de contacto intergrupal de los participantes con todas las variables de interés: estereotipos, prejuicio y amenaza. Sin embargo, solo algunas demostraron cierta asociación, como se muestra en la Tabla 2. Se encuentra que a mayor contacto con migrantes venezolanos, mayor es la posibilidad de calificarlos como conocidos, $r(115)$ $=.66, p<.01 ;$ amigos, $r(115)=.49, p<.01 ; 0$ compañeros de trabajo, $r(115)=.35, p<.01$. También, aumenta la percepción de similitud entre 
peruanos y venezolanos, $r(115)=.19, p<.05$, y se reduce el nivel de prejuicio manifiesto. A su vez, se encuentra que a mayor número de conocidos que sean migrantes venezolanos, se reducen los niveles de prejuicio manifiesto. Con respecto a la percepción de similitud entre ambos grupos, mientras mayor sea el parecido que el participante percibe entre peruanos $\mathrm{y}$ venezolanos, se reducen los niveles de prejuicio sutil, prejuicio manifiesto y amenaza exogrupal.

De acuerdo al primer objetivo de la investigación, se busca analizar la relación entre los estereotipos y el prejuicio. Como se muestra en la Tabla 3, se encuentra que mientras más estereotipos de competencia, calidez o moralidad presenten, menor es el prejuicio en sus expresiones sutil y manifiesto. Además, se encuentra que, de los tres tipos de estereotipia, la moralidad se asocia de manera inversa y más intensamente con el prejuicio en sus dimensiones sutil y manifiesto.
Los resultados que responden al segundo objetivo, analizar la mediación de la amenaza exogrupal en la relación entre estereotipos y el prejuicio ambivalente, se presentan en la Tabla 4. Se observa que a mayor amenaza percibida, mayor puntuación en ambas dimensiones del prejuicio; aunque, la amenaza se asocia inversamente a los estereotipos de moralidad, competencia y calidez. En específico, se encuentra que la amenaza simbólica se relaciona directamente con el prejuicio e inversamente con los estereotipos de moralidad, competencia y calidez. Por otro lado, la amenaza real se asocia directamente y más intensamente al prejuicio que la dimensión simbólica, al mismo tiempo que la magnitud de su relación con los estereotipos de moralidad, competencia y calidez es menor y de signo inverso. Cabe mencionar que existe una relación significativa entre ambas dimensiones de amenaza, $r(115)=.72, p<.01$.

Tabla 1

Descriptivos de los puntajes de estereotipos, prejuicio y amenaza

\begin{tabular}{lcccc}
\hline & \multicolumn{4}{c}{ Descriptivos } \\
\cline { 2 - 5 } & Min. & Max. & $M$ & $D E$ \\
\hline Estereotipos & 2 & 7 & 4.54 & 1.02 \\
$\quad$ Competencia & 2 & 7 & 4.63 & 1.07 \\
Calidez & 2 & 7 & 4.80 & 1.18 \\
$\quad$ Moralidad & 1 & 7 & 4.24 & 1.12 \\
Prejuicio & 1 & 5 & 2.78 & .74 \\
$\quad$ Sutil & 1 & 5 & 2.88 & .69 \\
$\quad$ Manifiesto & 1 & 5 & 2.63 & .94 \\
Amenaza exogrupal & 1 & 5 & 2.65 & 1.09 \\
$\quad$ Simbólica & 1 & 5 & 1.98 & 1.11 \\
$\quad$ Real & 1 & 5 & 2.98 & 1.19 \\
\hline
\end{tabular}


Tabla 2

Correlaciones entre datos de contacto intergrupal con estereotipos, prejuicio y amenaza

\begin{tabular}{cccccccc}
\hline & Contacto & Conocido & Vecino & $\begin{array}{c}\text { Compañero } \\
\text { de trabajo }\end{array}$ & Amigo & Familiar & $\begin{array}{c}\text { Similitud } \\
\text { intergrupal }\end{array}$ \\
\hline ECo & .084 & .056 & .073 & -.069 & .030 & -.029 & .037 \\
ECa & .089 & .076 & -.005 & -.083 & .055 & -.041 & .006 \\
EM & .110 & .124 & .091 & .037 & .099 & -.027 & .121 \\
PS & -.142 & -.176 & -.007 & -.144 & -.043 & -.029 & $-.376^{* *}$ \\
PM & $-.190^{*}$ & $-.229 *$ & .075 & -.179 & -.181 & -.050 & $-.259^{* *}$ \\
AS & -.092 & -.143 & -.082 & -.046 & -.077 & -.009 & $-.192^{*}$ \\
AR & -.007 & -.056 & .119 & .053 & .013 & .008 & $-.211^{*}$ \\
\hline
\end{tabular}

Nota: ${ }^{* *} p \leq .01 ;{ }^{*} p \leq .05$; ECo $=$ estereotipos de competencia; ECa $=$ estereotipos de calidez; EM = estereotipos de moralidad; $\mathrm{PS}=$ prejuicio sutil; $\mathrm{PM}=$ prejuicio manifiesto; $\mathrm{AS}=$ amenaza simbólica, $\mathrm{AR}=$ amenaza real.

Tabla 3

Correlaciones entre variables de estereotipos y prejuicio

\begin{tabular}{|c|c|c|c|c|c|}
\hline Variable & 1 & 2 & 3 & 4 & 5 \\
\hline 1. Estereotipos de competencia & 1 & & & & \\
\hline 2. Estereotipos de calidez & $.802^{* *}$ & 1 & & & \\
\hline 3. Estereotipos de moralidad & $.755^{* *}$ & $.701^{* *}$ & 1 & & \\
\hline 4. Prejuicio sutil & $-.425 * *$ & $-.316^{* *}$ & $-.606 * *$ & 1 & \\
\hline 5. Prejuicio manifiesto & $-.456 * *$ & $-.403^{* *}$ & $-.658 * *$ & $.745^{* *}$ & 1 \\
\hline
\end{tabular}

Nota: $* * p \leq .01 ; * p \leq .05$

\section{Tabla 4}

Correlaciones entre amenaza, estereotipos y prejuicio

\begin{tabular}{ccccccc}
\hline Variable & ECo & ECa & EM & PT & PS & PM \\
\hline AT & $-.419^{* *}$ & $-.409^{* *}$ & $-.515^{* *}$ & $.594^{* *}$ & $.529 * *$ & $.582^{* *}$ \\
AS & $-.429 * *$ & $-.421^{* *}$ & $-.502^{* *}$ & $.498^{* *}$ & $.437 * *$ & $.493^{* *}$ \\
AR & $-.376^{* *}$ & $-.367^{* *}$ & $-.474^{* *}$ & $.586^{* *}$ & $.523^{* *}$ & $.571^{* *}$ \\
\hline
\end{tabular}

Nota: $* * p \leq .01 ; * p \leq .05 ;$ AT = amenaza total; AS = amenaza simbólica; AR = amenaza real; ECo = estereotipos de competencia; $\mathrm{ECa}=$ estereotipos de calidez; $\mathrm{EM}$ = estereotipos de moralidad; $\mathrm{PT}$ = prejuicio total; $\mathrm{PS}=$ prejuicio sutil; $\mathrm{PM}=$ prejuicio manifiesto.

\section{Análisis de regresión}

Con la finalidad de analizar el efecto de la amenaza como variable mediadora en la relación entre los estereotipos hacia los migrantes venezolanos, como variable independiente, y el prejuicio de los ciudadanos peruanos, como variable dependiente, se han realizado análisis de regresión que pretenden medir la intensidad de la asociación entre variables para estimar la capacidad de predicción entre ellas. Estimamos la regresión lineal simple del prejuicio sutil sobre los estereotipos de competencia y se comprobó que están negativamente relacionados. Se encuentra que la pendiente $\beta=-.43$, $t(114)=-4.99, p<.001$ fue estadísticamente significativa y, por tanto, aceptamos la hipótesis de relación lineal entre estereotipos de competencia y 
prejuicio sutil. El valor de $R^{2}$ fue de .17 indicando que aproximadamente el $17 \%$ de la variabilidad del prejuicio sutil es explicado por los estereotipos de competencia.

A su vez, se probó la hipótesis de que los estereotipos de calidez y el prejuicio sutil están negativamente relacionados. Encontramos que la pendiente $\beta=-.32, t(114)=-3.54, p<.001$ fue significativa y se aceptó la hipótesis de relación lineal entre estereotipos de calidez y prejuicio sutil. El valor de $R^{2}$ fue de .09 indicando que aproximadamente el 9\% de la variabilidad del prejuicio sutil es explicado por los estereotipos de calidez. Además, se encuentra que los estereotipos de moralidad están negativamente relacionados con el prejuicio sutil, donde la pendiente $\beta=-.61, t(114)=-8.09, p<.001$ fue estadísticamente significativa y, por tanto, se aceptó la hipótesis de relación lineal entre estereotipos de moralidad y prejuicio sutil. El valor de $R^{2}$ fue de .32 indicando que aproximadamente el $32 \%$ de la variabilidad del prejuicio sutil es explicado por los estereotipos de moralidad. Con respecto al análisis de mediación de la variable de amenaza en la relación entre las dimensiones de estereotipos y el prejuicio sutil, se encontró que el coeficiente de regresión resulta significativamente menor en todos los casos. Esta diferencia se encuentra en la relación entre prejuicio sutil y estereotipos de competencia (Figura 1), estereotipos de calidez (Figura 2) y estereotipos de moralidad (Figura 3).

Figura 1

Modelo de mediación parcial de amenaza en la relación entre estereotipos de competencia y prejuicio sutil

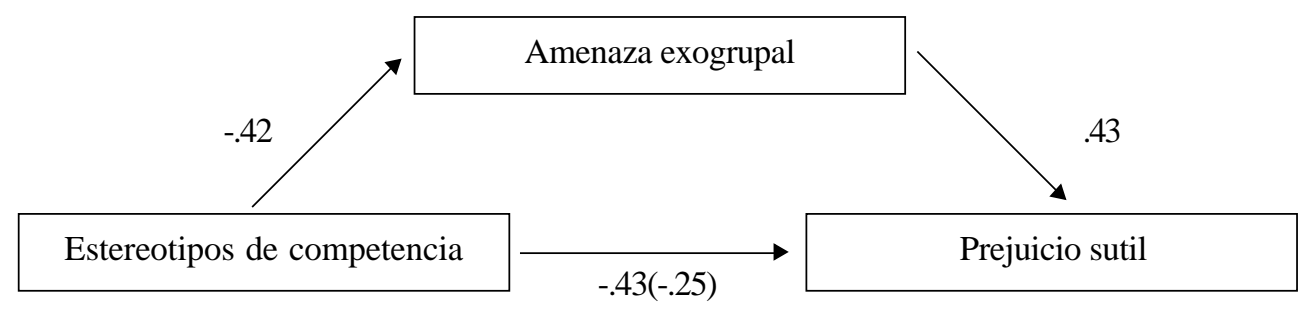

\section{Figura 2}

Modelo de mediación parcial de amenaza en la relación entre estereotipos de calidez y prejuicio sutil

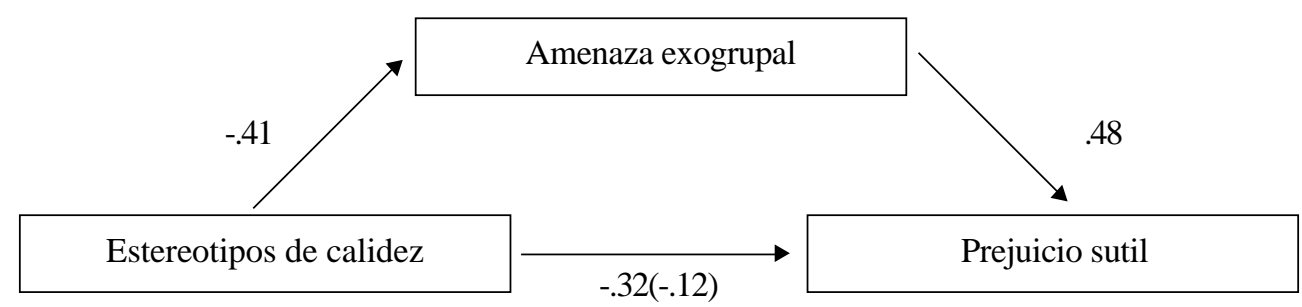




\section{Figura 3}

Modelo de mediación parcial de amenaza en la relación entre estereotipos de moralidad y prejuicio sutil

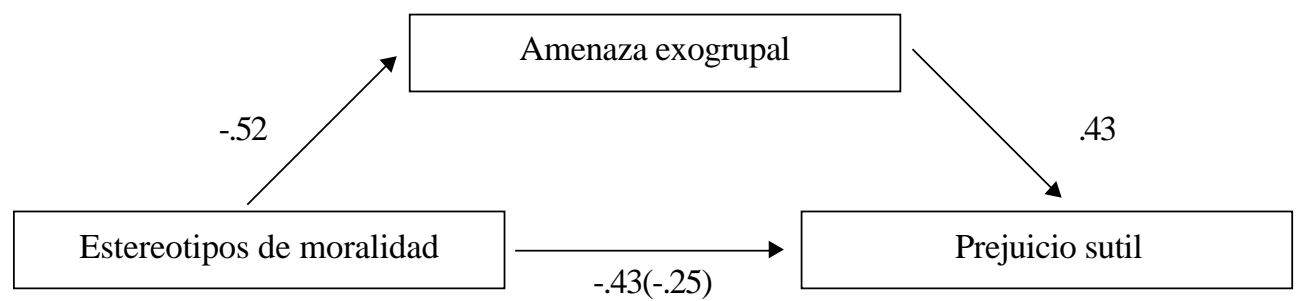

Por otro lado, estimamos la regresión lineal simple de los estereotipos de competencia sobre el prejuicio manifiesto y probamos la hipótesis de que están negativamente relacionados. Debido a que la pendiente $\beta=-.46, t(114)=-5.45, p<.001$ fue estadísticamente significativa, aceptamos la hipótesis de relación lineal entre estereotipos de competencia y prejuicio manifiesto. El valor de $R^{2}$ fue de .20 indicando que aproximadamente el $20 \%$ de la variabilidad del prejuicio manifiesto es explicado por los estereotipos de competencia. También, se estimó la regresión lineal del prejuicio manifiesto sobre los estereotipos de calidez, encontrando que están negativamente relacionados. La pendiente $\beta=-.40$, $t(114)=-4.68, p<.001$ fue estadísticamente significativa y, por ello, aceptamos la hipótesis de relación lineal entre estereotipos de calidez y prejuicio manifiesto. El valor de $R^{2}$ fue de .15 , de modo que aproximadamente el $15 \%$ de la variabilidad del prejuicio manifiesto es explicado por los estereotipos de calidez.

En cuanto al análisis de regresión lineal entre los estereotipos de moralidad y el prejuicio manifiesto, se encontró que están negativamente relacionados. Debido a que se observa que la pendiente $\beta=-.66$, $t(114)=-9.29, p<.001$ fue estadísticamente significativa, aceptamos la hipótesis de relación lineal entre estereotipos de moralidad y prejuicio manifiesto.
Se observa que el valor de $R^{2}$ fue de .43 indicando que aproximadamente el $43 \%$ de la variabilidad del prejuicio manifiesto es explicado por los estereotipos de moralidad. A su vez, se realizó un análisis de mediación de la variable de amenaza en la relación entre las dimensiones de estereotipos y el prejuicio manifiesto, donde se encontró que el coeficiente de regresión resulta ser significativamente menor. Esta diferencia se observa en la relación entre prejuicio manifiesto y estereotipos de competencia (Figura 4), estereotipos de calidez (Figura 5) y estereotipos de moralidad (Figura 6).

Para finalizar, se realizó el mismo análisis con los puntajes totales de las variables de interés donde comprobamos la hipótesis de que los estereotipos están negativamente relacionados con el prejuicio. Se observa que la pendiente $\beta=-.57, t(114)=-7.29, p$ $<.001$ fue estadísticamente significativa y, por tanto, aceptamos la hipótesis de relación lineal entre estereotipos y prejuicio. El valor de $R^{2}$ fue de .31 , por lo cual, se calcula que aproximadamente el 31\% de la variabilidad del prejuicio es explicado por los estereotipos. Con respecto al análisis de mediación, se encontró que el coeficiente de regresión de la relación entre estereotipos y prejuicio disminuye con la incorporación de la variable de amenaza (Figura 7). 


\section{Figura 4}

Modelo de mediación parcial de amenaza en la relación entre estereotipos de competencia y prejuicio manifiesto

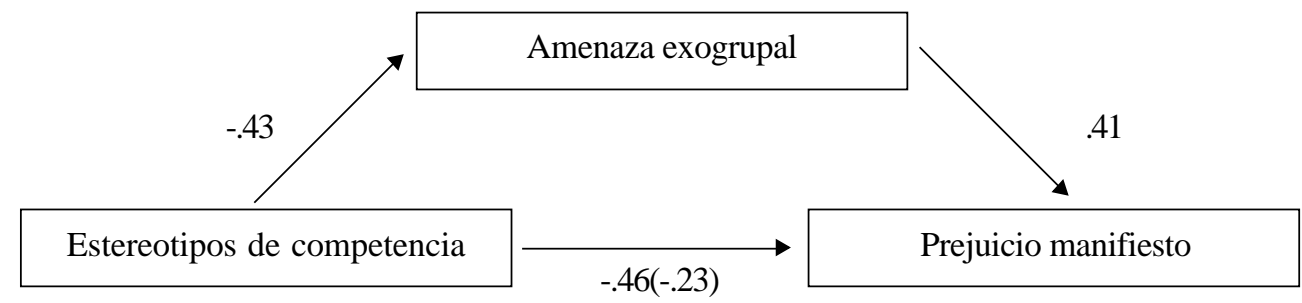

\section{Figura 5}

Modelo de mediación parcial de amenaza en la relación entre estereotipos de calidez y prejuicio manifiesto

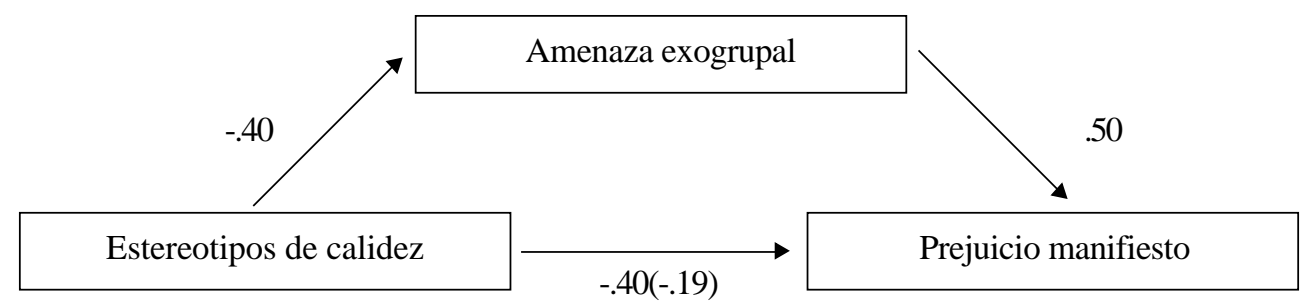

\section{Figura 6}

Modelo de mediación parcial de amenaza en la relación entre estereotipos de moralidad y prejuicio manifiesto

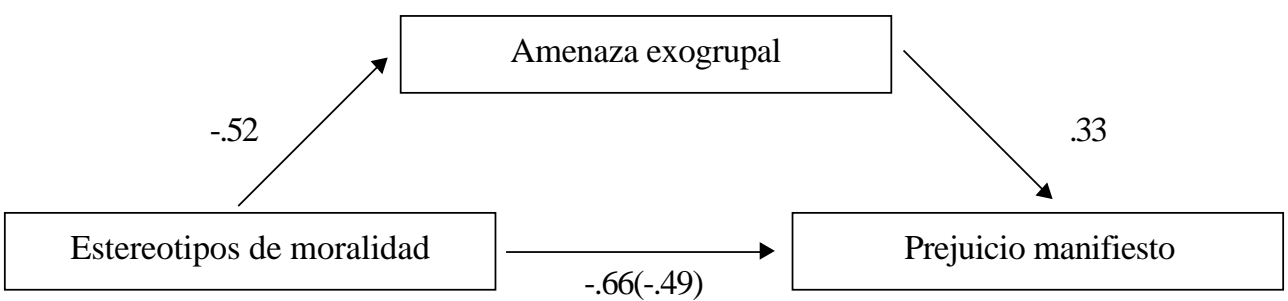




\section{Figura 7}

Modelo de mediación parcial de amenaza en la relación entre estereotipos y prejuicio

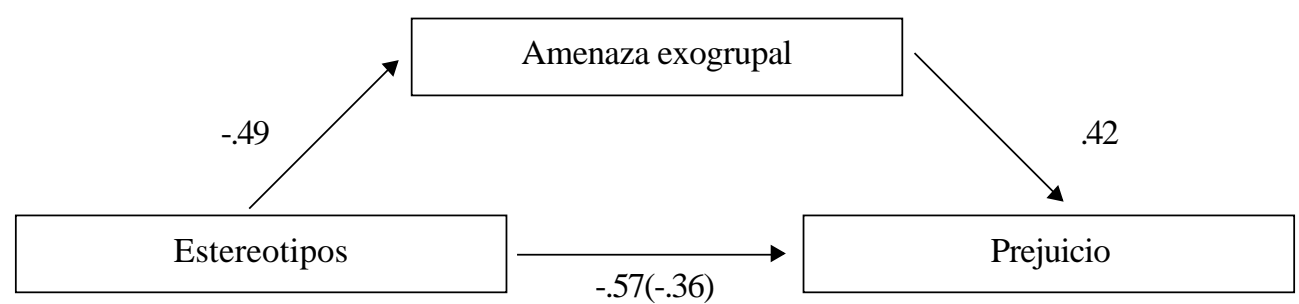

\section{Discusión}

La formación de estereotipos constituye un proceso cognitivo a partir del cual se atribuyen ciertas características a las personas sobre la base de su pertenencia a categorías o grupos sociales (Smith, 2006). Los resultados muestran que los peruanos que participaron en esta investigación mantienen una representación moderadamente positiva sobre los migrantes venezolanos, siendo saliente, a nivel descriptivo, la representación de calidez. Por lo cual, se situaría a los venezolanos como un grupo de bajo estatus con algunos rasgos de sociabilidad, pero no necesariamente representados en términos de competencia o moralidad y, por lo tanto, no necesariamente confiables desde la perspectiva de los participantes (Espinosa et al., 2016; Fiske et al., 2002; Moscatelli et al., 2018).

En cuanto a la relación entre los estereotipos y el prejuicio, consistentemente con lo esperado, mayores puntuaciones en las dimensiones estereotípicas descritas en el estudio tienden a relacionarse inversamente con las expresiones, sutil y manifiesta, del prejuicio. Lo cual sugiere que representaciones más positivas del exogrupo tenderán a mitigar las valoraciones prejuiciosas sobre el mismo (Allport, 1954; Allport, 2000; Hainmueller \& Hopkins, 2014). De manera más específica, aunque la prevalencia de los aspectos positivos de las tres dimensiones estereotípicas estudiadas tiende a mitigar las expresiones del prejuicio, es importante resaltar que las relaciones de mayor magnitud son las existentes entre la dimensión de moralidad y las dimensiones del prejuicio ambivalente. Dichas relaciones ponen en evidencia que, los atributos y características que permiten construir la representación de un grupo en términos de confianza, honestidad y una conducta socialmente responsable, son, quizás, los elementos más importantes en la reducción del prejuicio (Fiske, 2000; Fiske et al., 2002; Yzerbyt, 2016).

Bajo esta misma lógica, la dimensión estereotípica de competencia vinculada a atributos y características de agencia, instrumentalismo y laboriosidad, también contribuye a mitigar el prejuicio. Ello en la medida en que la competencia comprende una serie de atributos que se asociarían a un alto estatus del grupo que la representa (Brambilla et al., 2012; Phalet \& Poppe, 1997; Wojciszke, 1994). Finalmente, la dimensión estereotípica de calidez que comprende una representación del exogrupo como sociable y simpático, sigue la misma tendencia en la reducción del prejuicio. Sin embargo, en este caso el tamaño del efecto de las relaciones es menor al de las dimensiones de moralidad y competencia. Así mismo, la magnitud del efecto de la relación de calidez es mayor cuando se vincula con la dimensión manifiesta, en comparación con la dimensión sutil del prejuicio. Esto podría relacionarse con el hecho de que es justamente la calidez, la dimensión sobre la que se argumenta la construcción de una expresión encubierta del prejuicio, que tal como lo muestra el resultado no es abiertamente hostil. En 
paralelo, no necesariamente se reconocen elementos de competencia o moralidad en el exogrupo, tornándose un correlato de representaciones prejuiciosas benevolentes, donde la evaluación del endogrupo hacia el exogrupo no está enmarcada en un rechazo abierto, sino en la asunción de una supuesta inferioridad o subordinación exogrupal, a pesar de que sus miembros puedan ser percibidos como sociables y simpáticos (Carmona-Halty et al., 2018; Muller et al., 2017).

Los resultados a nivel correlacional también evidencian que el contacto con inmigrantes venezolanos, especialmente con conocidos, se relaciona de manera inversa con el prejuicio manifiesto. Sumado a esto, la percepción de similitud de los venezolanos con los miembros del endogrupo nacional, también se asocia inversamente con el prejuicio. Al respecto, se ha establecido que el contacto intergrupal a partir de la colaboración promueve el acercamiento de los grupos sociales; y así, se abren posibilidades de un conocimiento mutuo menos superficial. A pesar de ello, la evidencia psicosocial sugiere que, en ocasiones, la cooperación intergrupal no resulta suficiente para generalizar representaciones y evaluaciones positivas de los grupos; se considera que es mejor que los grupos se acerquen e interactúen, antes que eviten el contacto entre sí, pues dichas aproximaciones ayudan a reforzar la idea de que los grupos no son diferentes o que las diferencias que pudieran presentar no son intratables o irreconciliables (Hewstone \& Greenland, 2000). En otras palabras, la interacción entre peruanos e inmigrantes provenientes de Venezuela podría reducir el rechazo de dicho colectivo, de modo que las valoraciones negativas sostenidas por sistemas de creencias racistas y segregacionistas podrían ser cuestionadas y abiertamente denunciadas.

En esta línea, podemos basarnos en la Teoría de Contacto Intergrupal de Allport (1954) para proponer las condiciones necesarias para promover un contacto intergrupal positivo que pueda acercar a los peruanos al grupo de migrantes venezolanos creando redes de contacto que disminuyan el prejuicio y la discriminación. Dicho autor identifica cuatro condiciones indispensables para el diseño de estos acercamientos: la igualdad de estatus de los participantes en la interacción, el planteamiento de objetivos comunes, una visión cooperativa de los grupos, y el apoyo institucional como una alternativa de mediación en posibles conflictos existentes entre los grupos. Esta última condición se refiere a las normas, sanciones y demás regulaciones que habiliten una relación adecuada; sin embargo, en el contexto peruano, los esfuerzos institucionales por ejecutar acciones o estrategias con estas características son pocos o inexistentes. Como resultado, la interacción entre ambos grupos resulta ser cada vez más defectuosa y distante.

Un problema derivado de condiciones de contacto deficientes, se observa en los resultados del estudio de Arana y Espinosa (2020) con migrantes venezolanos, quienes, en su proceso de aculturación, reportan una fuerte adhesión a estrategias de marginalización. Y es que las experiencias de discriminación más frecuentemente reportadas por estos, se relacionan con la dificultad percibida para establecer relaciones interpersonales positivas con peruanos, lo que dificulta su integración a la sociedad. Asimismo, estos aspectos de discriminación se relacionan con las dificultades para el ejercicio de la ciudadanía de los venezolanos en el Perú, produciendo una sensación de exclusión por parte de una sociedad que no le reconoce sus derechos. Por lo anteriormente descrito, los autores mencionan que existe una alta probabilidad de que los venezolanos reaccionen alienándose y desarrollen desconfianza hacia los peruanos, lo que explicaría también la prevalencia de la marginalización como estrategia aculturativa predominante (Arana \& Espinosa, 2020).

El contacto intergrupal es importante en la medida en que, como se ha mencionado previamente, los participantes que reportan un mayor contacto con migrantes venezolanos tienden a percibirlos más similares también. Siendo esta percepción de similitud 
intergrupal, una forma de favorecer la estereotipia y el afecto positivo entre grupos. Así, de acuerdo a lo propuesto por Allport (1954), se podría facilitar el desarrollo de relaciones cercanas mediante el descubrimiento de similitudes entre los miembros de ambos grupos para luego desarrollar un afecto positivo mutuo. En la interacción, los participantes irán identificando información que contradiga los estereotipos negativos que, hasta ese momento, mantienen sobre el otro.

Es importante resaltar que el contacto debe ser próximo para que inhiba el prejuicio. De modo que, el trato directo con miembros del exogrupo de migrantes venezolanos favorezca la valoración sobre los mismos, en tanto la relación sea de conocidos o más cercana. Sin embargo, como se ha observado, este acercamiento dependerá del ajuste mutuo que experimentan ambos grupos en la convivencia. De acuerdo a la Teoría de Aculturación (Berry, 1990), se produce un cambio cultural para el grupo dominante y el grupo subordinado como efecto del intercambio intercultural, debido a que el abandono del país de origen y la llegada a una sociedad de acogida supone el desarrollo de una serie de efectos psicosociales. A nivel grupal, principalmente, supone cambios en las identidades, comportamientos, valores, estructuras sociales e institucionales de ambas sociedades. Mientras que, a nivel individual, las transformaciones se producen en la cognición, motivación, actitudes y conductas de las personas implicadas en el proceso (Berry, 1990).

Por otro lado, además del contacto intergrupal, la ideología puede inhibir el prejuicio, en tanto se asuma la convivencia con dicho grupo en términos de cooperación, puesto que una ideología conservadora o autoritaria puede motivar la competencia y generar un mayor distanciamiento o rechazo social. Existen antecedentes que sugieren que las ideologías conservadoras se relacionan negativamente con las representaciones estereotípicas y la valoración exogrupal (Valencia-Moya et al., 2018). En este sentido, la Teoría del Conflicto Realista sostiene que la hostilidad intergrupal emerge de la competencia entre los grupos por recursos socialmente valorados y aparentemente escasos como poder, prestigio y bienes materiales (Smith, 2006). Así, las motivaciones individuales no son suficientes para explicar la hostilidad generalizada, por lo tanto, se cuestionan las relaciones entre los grupos para identificar las características y normas que reproducen relaciones desiguales.

Además, se encuentra que la amenaza es una variable mediadora en la relación entre estereotipos y prejuicio. Según la Teoría de la Amenaza Integrada de Stephan y Stephan (2000), la percepción de amenaza no necesariamente responde a criterios objetivos de peligro, lo importante es la realidad psicológica. Dicha teoría argumenta que existen distintos tipos de amenazas, las cuales pueden atentar contra la integridad del grupo, la posición de privilegio y la reproducción cultural del endogrupo. Cuando las posiciones de los grupos se ven amenazadas, las personas sienten una mayor necesidad de aferrarse a sus categorías sociales y aumenta la hostilidad hacia los exogrupos (Smith, 2006). En otras palabras, una elevada percepción de amenaza representa un problema para la inclusión social.

En este sentido, esta investigación busca aportar a la investigación de las relaciones entre amenaza, prejuicio y estereotipo, las cuales suelen ser desarrolladas en otros contextos, y pocas veces aplicadas a la investigación en Latinoamérica. En la actualidad, se están desarrollando aportes, desde un enfoque psicosocial, que buscan reconocer el efecto de la migración masiva de venezolanos al contexto peruano. Un referente directo de esta investigación tuvo como resultado que existe una relación entre ideología autoritaria y prejuicios frente a los migrantes, justificada en la preservación del endogrupo peruano. Además, sostiene que la representación positiva de los peruanos se relaciona directamente con una valoración positiva de los venezolanos (Ferreyros, 2019).

En conclusión, la emigración internacional masiva es un fenómeno conocido para el país, sin embargo, 
lo novedoso es que Perú sea la sociedad receptora de inmigrantes venezolanos. Estudiar las consecuencias de la interacción de grupos culturalmente distintos, permite identificar vías de intervención pertinentes para lograr una sociedad más inclusiva. La xenofobia encuentra su origen en los prejuicios etnocéntricos, indistintamente de las posibles experiencias negativas con los inmigrantes (Alaminos et al., 2010). La representación negativa y el prejuicio surge pensando en el colectivo de inmigrantes, a partir de la desinformación y la exposición mediática de titulares que los retratan de forma negativa y dañina para la estabilidad y seguridad nacional (Schlueter \& Davidov, 2013). En consecuencia, refuerza y contribuye a que se mantengan las sobregeneralizaciones sobre los mismos a partir de las cuales se pretende justificar los actos y expresiones xenofóbicas. En suma, identificar las redes de contacto, las actitudes y valoraciones sobre el exogrupo, la representación positiva y el grado de amenaza percibido puede contribuir a planificar sobre el contexto donde se puede intervenir para motivar la integración de los inmigrantes venezolanos.

Con respecto a las limitaciones del estudio, la cantidad de participantes y las características socioeconómicas de los mismos (por ejemplo, grado de instrucción, nivel socioeconómico y/u ocupación) no permiten realizar análisis comparativos con respecto a los grupos que compiten directamente con los inmigrantes venezolanos por el acceso a puestos de trabajo, educación o servicios públicos de salud. Además, la cantidad de participantes por sexo no fue proporcional, aunque dentro de los objetivos de la investigación no se considera realizar un análisis de ese tipo, es importante identificar una mayor cantidad y variedad de participantes con la finalidad de explorar las diferencias por grupo en la construcción de las representaciones y prejuicios sobre los migrantes venezolanos.

Finalmente, se recomienda generar estrategias que permitan el contacto directo entre grupos, a partir del cual se promueva la integración social de minorías inmigrantes. La desigualdad social surge en relación al poder, entendido como el control que una persona o grupo tiene sobre otro, y determinado por la superioridad de recursos económicos que un grupo tiene respecto a los subordinados a él (García-Castro, 2010). En la exploración de cómo se construyen los estereotipos y valoraciones que legitiman la desigualdad, se identifican los sistemas de creencias por cuestionar y modificar. Las autoridades deberían enfocar sus esfuerzos en enfatizar la importancia de los programas sociales y educativos, los cuales tengan como enfoque los derechos humanos y la inclusión social. Resulta especialmente importante que estas iniciativas sean impulsadas por las instituciones y autoridades gubernamentales, debido a que la condición esencial para propiciar relaciones intergrupales solidarias está dada por la estructura social que determina las relaciones entre los grupos y sus miembros. Por último, se recomienda cuestionar el rol de los medios de comunicación en la producción y el reforzamiento de representaciones negativas sobre el migrante latinoamericano, pues usualmente se presenta al mismo como ilegal o destructivo, reforzando los estereotipos negativos.

\section{Conflicto de intereses}

Los autores refieren que no existe ningún tipo de conflicto de intereses.

\section{Responsabilidad ética}

Se informó a los participantes de los criterios de confidencialidad, anonimato y voluntariedad. Todos brindaron su consentimiento antes de completar los formularios. Los datos fueron analizados colectivamente y solo con fines estrictamente académicos. El proceso de devolución de resultados fue realizado al finalizar la investigación, solo a los participantes que brindaron un correo electrónico de contacto.

\section{Contribución de autoría}

MGR, AEP: ambos autores participaron en todo el proceso de investigación. 


\section{Referencias}

Alaminos, A., López, C., \& Santacreu, O. (2010). Etnocentrismo, xenofobia y migraciones internacionales en una perspectiva comparada. Convergencia, 17(53), 91-124.

Alayo, F. (2019, 29 de abril). El 67\% de limeños no está de acuerdo con la inmigración venezolana al Perú. El Comercio. https://cutt.ly/mjRLLPb

Allport, G. W. (1954). The Nature of Prejudice. AddisonWesley Publishing Company.

Allport, G. W. (2000). The Nature of Prejudice. En Stereotypes and Prejudice. Psychology Press.

Arana, M., \& Espinosa, A. (2020). Estilos aculturativos y su relación con la discriminación percibida y la experiencia de ciudadanía subjetiva en migrantes venezolanos en el Perú. Inclusão Social, 13(2), 135148. http://revista.ibict.br/inclusao/article/view/5516

Atwell, A., Ma, R., Chien, H. Y., \& Mastro, D. (2018). Cultivating Intergroup Emotions: An Intergroup Threat Theory Approach. Mass Communication and Society, 21(2), 178-197. https://doi.org/10.1080/15205436.2 017.1381262

Berry, J. W. (1990). Psychology of Acculturation. En J. Berman (Ed.), Cross Cultural Perspectives: Nebraska Symposium of Motivation (pp. 457-488). University of Nebraska Press.

Brambilla, M., Sacchi, S., Rusconi, P., Cherubini, P., \& Yzerbyt, V. Y. (2012). You Want to Give a Good Impression? Be Honest! Moral Traits Dominate Group Impression Formation. British Journal of Social Psychology, 51(1), 149-166. https://doi.org/10.1111/ j.2044-8309.2010.02011.x

Carmona-Halty, M., \& Navas, M. (2016). Análisis Psicométrico de la Escala de Percepción de Amenaza Exogrupal (EPAE) en una muestra chilena. Interciencia, 41(11), 788-794.

Carmona-Halty, M., Navas, M., \& Rojas-Paz, P. (2018). Percepción de amenaza exogrupal, contacto intergrupal y prejuicio afectivo hacia colectivos migrantes latinoamericanos residentes en Chile. Interciencia: Revista de Ciencia y Tecnología de América, 43(1), 23-27.
Castles, S. (2010). Migración irregular: causas, tipos y dimensiones regionales. Migración y Desarrollo, 8(15), 49-80.

Castro, V. S. (2006). La psicología social de las relaciones intergrupales: modelos e hipótesis. Actualidades en Psicología, 20(107), 45-71.

Civalero, L., Alonso, D., \& Brussino, S. (2019). Evaluación del prejuicio hacia inmigrantes: adaptación argentina de la escala de prejuicio sutil y manifiesto. Ciencias Psicológicas, 13(1), 119-133. http://dx.doi.org/10.22235/ cp.v13i1.1814

Contreras-Ibáñez, C. C., \& Saldívar, A. (2018). Sobre la relación entre la identificación con el estereotipo nacional mexicano y las actitudes hacia los inmigrantes. Polis, 14(2), 39-69.

Espinosa, A., Acosta, Y., Valencia, J., Vera, A., da Silva, A. S., Romero, J. C., \& Beramendi, M. (2016). Calidez, competencia, moralidad y nacionalismo ideal como dimensiones autoestereotípicas del autoconcepto nacional en seis países de Latinoamérica. Avances en Psicología Latinoamericana, 34(2), 395-414. https:// doi.org/10.12804/apl34.2.2016.12

Ferreyros, J. A. (2019). Bases motivacionales del prejuicio a inmigrantes venezolanos en el Perú [tesis de licenciatura, Pontificia Universidad Católica del Perú]. Repositorio. http://hdl.handle.net/20.500.12404/15336

Fiske, S. (2000). Sterotyping, Prejudice, and Discrimination at the Seam Between the Centuries: Evolution, Culture, Mind, and Brain. European Journal of Social Psychology, 30(3), 299-322. https://doi.org/10.1002/ (SICI)1099-0992(200005/06)30:3<299::AIDEJSP2>3.0.CO;2-F

Fiske, S. T., Cuddy, A. J., Glick, P., \& Xu, J. (2002). A Model of (Often Mixed) Stereotype Content: Competence and Warmth Respectively Follow from Perceived Status and Competition. Journal of Personality and Social Psychology, 82(6), 878-902. https://doi.org/10.1037/ 0022-3514.82.6.878

García-Castro, J. D. (2010). Ideología de la desigualdad: Análisis de la investigación empírica en psicología social. Revista Electrónica de Psicología Política, 8(24), 67-87. http://hdl.handle.net/10669/82023 
Granados, G. (2019, 26 de noviembre). Migración venezolana: 4.500 kilómetros entre el abandono y la oportunidad. Banco Mundial. https://cutt.ly/1jRKsDd

Hainmueller, J., \& Hopkins, D. J. (2014). Public Attitudes Toward Immigration. Annual Review of Political Science, 17, 225-249. https://doi.org/10.1146/annurevpolisci-102512-194818

Hewstone, M., \& Greenland, K. (2000). Intergroup Conflict. International Journal of Psychology, 35(2), 136-144. https://doi.org/10.1080/002075900399439

Instituto de Opinión Pública - IOP. (2018). Creencias y actitudes hacia la inmigración en el Perú. Estado de la Opinión Pública, 156, 1-16. http://repositorio.pucp. edu.pe/index/handle/123456789/133523

López-Rodríguez, L., Cuadrado, I., \& Navas, M. (2013). Aplicación extendida del Modelo del Contenido de los Estereotipos (MCE) hacia tres grupos de inmigrantes en España. Estudios de Psicología, 34(2), 197-208.

López-Rodríguez, L., Vázquez, A., Cuadrado, I., Brambilla, M., Rodrigo, M., \& Dovidio, J. F. (2020). Immigration: An Invasion or an Opportunity to the Country. The Effect of Real News Frames of Immigration on Ethnic Attitudes. International Journal of Social Psychology, 35(3), 1-40. https://doi.org/10.1080/02134748.2020. 1783834

Lotero-Echeverri, G., \& Pérez-Rodríguez, M. A. (2019). Migraciones en la sociedad contemporánea: Correlación entre migración y desarrollo. Retos, Revista de Ciencias de la Administración y Economía, 9(17), 145-159. https://doi.org/10.17163/ret.n17.2019.09

Mackie, D. M., Devos, T., \& Smith, E. R. (2000). Intergroup Emotions: Explaining Offensive Action Tendencies in an Intergroup Context. Journal of Personality and Social Psychology, 79(4), 602-616. https://doi.org/ 10.1037/0022-3514.79.4.602

Martínez, C. (1996). Análisis Psicosocial del Prejuicio. Síntesis Psicología.

Martínez, M. F., Calzado, V., \& Martínez, J. (2011). La orientación a la dominancia social y la identidad nacional en las actitudes hacia las políticas migratorias: el papel mediador de la amenaza percibida y el tamaño del exogrupo. Revista de Psicología Social, 26(1), 2132. https://doi.org/10.1174/021347411794078408
Meneses, M. (2012). La migración internacional de peruanos a Europa. Investigaciones Sociales, 16(29), 285-290. https://doi.org/10.15381/is.v16i29.7813

Mera, M. J., Martínez, C., \& Costalat-Founeau, A. M. (2014). Dinámicas identitarias en procesos de transición psicosocial: Adolescencia y migración. Estudio de caso. Migraciones internacionales, 7(3), 221-248.

Moscatelli, S., Menegatti, M., Albarello, F., Pratto, F., \& Rubini, M. (2018). Can we Identify with a Nation Low in Morality? The Heavy Weight of (Im)Morality in International Comparison. Political Psychology, 40(1), 93-110. https://doi.org/10.1111/pops.12504

Muller, M., Ungaretti, J., \& Etchezahar, E. (2017). Validación argentina de la Escala de Prejuicio Sutil y Manifiesto hacia villeros. Revista de Psicología (Santiago), 26(1), 1-13. http://dx.doi.org/10.5354/0719-0581.2017.46204

Navas, M., Cuadrado, I., \& López-Rodríguez, L. (2012). Fiabilidad y evidencias de validez de la Escala de Percepción de Amenaza Exogrupal (EPAE). Psicothema, 24(3), 477-482.

Olson, M., \& Zabel, K. (2016). Measures of prejudice. En T. Nelson (Ed.), Handbook of prejudice, stereotyping, and discrimination (pp. 175-211). Psychology Press.

Organización Internacional para las Migraciones - OIM. (2018). Informe sobre las migraciones en el mundo 2018. Organización Internacional para las Migraciones.

Palacio, J., Ramos-Vidal, I., Llinas-Solano, H., Doria-Zapata, A., \& Nogueda-Cadena, K. (2020). Adaptación y validación de la Escala de Prejuicio Sutil y Manifiesto hacia inmigrantes venezolanos en una muestra colombiana. Revista de Psicología, 38(1), 197-222. https://doi.org/10.18800/psico.202001.008

Perea, H., Belapatiño, V., Grippa, F., Mendoza, I., \& Vega, H. (2019). Inmigración venezolana a Perú: características e impactos macroeconómicos. BBVA Research Perú. https://www.bbvaresearch.com/ publicaciones/inmigracion-venezolana-a-perucaracteristicas-e-impactos-macroeconomicos/

Pereira, C., Vala, J., \& Leyens, J. P. (2009). From InfraHumanization to Discrimination: The Mediation of Symbolic Threat Needs Egalitarian Norms. Journal of Experimental Social Psychology, 45(2), 336-344. https://doi.org/10.1016/j.jesp.2008.10.010 
Pettigrew, T. F., \& Meertens, R. W. (1995). Subtle and Blatant Prejudice in Western Europe. European Journal of Social Psychology, 25(1), 57-75. https:// doi.org/10.1002/ejsp.2420250106

Phalet, K., \& Poppe, E. (1997). Competence and Morality Dimensions of National and Ethnic Stereotypes: A Study in Six Eastern European Countries. European Journal of Social Psychology, 27(6), 703-723. https:// doi.org/10.1002/(S ICI)1099-0992(199711/ 12)27:6<703::AID-EJSP841>3.0.CO;2-K

Ríos, K., Sosa, N., \& Osborn, H. (2018). An Experimental Approach to Intergroup Threat Theory: Manipulations, Moderators, and Consequences of Realistic vs. Symbolic Threat. European Review of Social Psychology, 29(1), 212-255. https://doi.org/10.1080/ 10463283.2018.1537049

Rueda, J., \& Navas, M. (1996). Hacia una evaluación de las nuevas formas del prejuicio racial: las actitudes sutiles del racismo. Revista de Psicología Social, 11(2), 131-150.

Schlueter, E., \& Davidov, E. (2013). Contextual Sources of Perceived Group Threat: Negative Immigration-Related News Reports, Immigrant Group Size and their Interaction, Spain 1996-2007. European Sociological Review, 29(2), 179-191. https://doi.org/10.1093/esr/ jcr054

Sherman, J. W. (1996). Development and mental representation of stereotypes. Journal of Personality and Social Psychology, 70(6), 1126-1141. https:// doi.org/10.1037/0022-3514.70.6.1126

Smith, V. (2006). La psicología social de las relaciones intergrupales: modelos e hipótesis. Actualidades en Psicología, 20(107), 45-71.

Stangor, C. (2016). The Study of Stereotyping, Prejudice, and Discrimination within Social Psychology: A Quick History of Theory and Research. En T. Nelson (Ed.), Handbook of Prejudice, Stereotyping, and Discrimination (2. ${ }^{\text {a }}$ ed., pp. 3-27). Psychology Press
Stephan, W. S., \& Stephan C. W. (2000). An Integrated Threat Theory of Prejudice. En S. Oskamp (Ed.), Reducing Prejudice and Discrimination (pp. 23-45). Lawrence Erlbaum Associates Publishers.

Stephan, W. G., Ybarra, O., \& Bachman, G. (1999). Prejudice Toward Immigrants. Journal of Applied Social Psychology, 29(11), 2221-2237. https://doi.org/ 10.1111/j.1559-1816.1999.tb00107.x

Superintendencia Nacional de Migraciones - SNM. (2018). Presencia de inmigrantes en situaciones de vulnerabilidad en el Perú. Estudio preliminar realizado desde una perspectiva de género. Superintendencia Nacional de Migraciones.

Valencia-Moya, J., Espinosa, A., Jiménez, V., \& Romero, J. C. (2018). Ideología, identidad nacional, representaciones estereotípicas y valoración exogrupal: un estudio sobre las relaciones entre Perú y Chile. Interciencia, 43(5), 304-312.

Willis-Esqueda, C., Delgado, R. H., \& Pedroza, K. (2017). Patriotism and the Impact on Perceived Threat and Immigration Attitudes. The Journal of Social Psychology, 157(1), 114-125. https://doi.org/10.1080/ 00224545.2016 .1184125

Wlodarczyk, A., Basabe, N., \& Bobowik, M. (2014). The Perception of Realistic and Symbolic Threat and its Influence on Prejudice, Ingroup Favouritism and Prosocial Response: The Native Population in the Face of Immigration. Revista de Psicología Social, 29(1), 60-89. https://doi.org/10.1080/02134748.2013.878574

Wojciszke, B. (1994). Multiple Meanings of Behavior: Construing Actions in Terms of Competence or Morality. Journal of Personality and Social Psychology, 67(2), 222-232. https://doi.org/10.1037/ 0022-3514.67.2.222

Yzerbyt, V. (2016). Intergroup Stereotyping. Current Opinion in Psychology, 11, 90-95. https://doi.org/ 10.1016/j.copsyc.2016.06.009 


\section{Milagros Gomez Robinson}

Pontificia Universidad Católica del Perú.

Bachiller en Psicología por la Pontificia Universidad Católica del Perú. Miembro del Grupo de Psicología Política y Social (GPPSPUCP).

ORCID: https://orcid.org/0000-0002-7363-0500

Autora corresponsal: milagros.gomez@pucp.pe

Agustín Espinosa

Pontificia Universidad Católica del Perú.

Doctor en Psicología Social por la Universidad del País Vasco y licenciado en Psicología Social por la Pontificia Universidad Católica del Perú (PUCP). Actualmente es profesor principal del Departamento Académico de Psicología de la PUCP y coordinador del Grupo de Psicología Política y Social (GPPS-PUCP).

ORCID: https://orcid.org/0000-0002-2275-5792

agustin.espinosa@pucp.pe 